\title{
Intraocular Pressure after Implantation of the Visian Implantable Collamer Lens With CentraFLOW Without Iridotomy
}

\author{
ALEJANDRO HIGUERAS-ESTEBAN, AMANDA ORTIZ-GOMARIZ, RAMÓN GUTIÉRREZ-ORTEGA, \\ CÉSAR VILLA-COLLAR, JUAN P. ABAD-MONTES, PAULO FERNANDES, AND JOSÉ M. GONZÁLEZ-MÉIJOME
}

- PURPOSE: To compare the intraocular pressure (IOP) values during a 3-month period after implantation of the new Visian Implantable Collamer Lens (ICL; STAAR Surgical Company, Nidau, Switzerland) V4c design with CentraFLOW technology without iridotomy using a standard procedure followed by implantation of the conventional ICL V4b model.

- DESIGN: Retrospective cohort study.

- METHODS: This study included 17 eyes implanted with the ICL V4b model and 18 eyes implanted with the ICL $\mathrm{V} 4 \mathrm{c}$ model. The mean preoperative spherical equivalent refractions were $-7.48 \pm 5.00$ diopters and $-8.66 \pm$ 4.2 diopters, respectively. We compared the bestcorrected distance visual acuity before surgery with the uncorrected distance visual acuity after surgery. The intraocular pressure (IOP) was measured 1 week, 1 month, and 3 months after surgery. The central vault at 3 months was measured using optical coherence tomography.

- RESULTS: Three months after surgery, the mean uncorrected distance visual acuities were $-0.09 \pm 0.12$ logarithm of the minimal angle of resolution units with the $\mathrm{V} 4 \mathrm{~b}$ and $-0.07 \pm 0.11$ logarithm of the minimal angle of resolution units with the $\mathrm{V} 4 \mathrm{c}$. The mean distances between the ICL and the anterior crystalline lens surface were $557 \pm 224 \mu \mathrm{m}$ and $528 \pm 268 \mu \mathrm{m}$ for the $\mathrm{V} 4 \mathrm{~b}$ and V4c, respectively $(P=.73)$. After 1 week and 1 month, the mean IOPs were 13.7 and $13.3 \mathrm{~mm} \mathrm{Hg}$ and 14.7 and $15.1 \mathrm{~mm} \mathrm{Hg}$, respectively. There were no significant differences in IOP within or between groups during the follow-up period ( $P>.05$, for all comparisons).

- CONClusions: The new ICL with the CentraFLOW design seems to provide similar results as its predecessors for the correction of moderate to high myopia and maintenance of safe IOP levels without iridotomy. (Am J Ophthalmol 2013;156:800-805. (C) 2013 by Elsevier Inc. All rights reserved.)

\section{Accepted for publication May 16, 2013}

From the Department of Optics and Optometry, Universidad Europea de Madrid, Madrid, Spain (A.H.-E., A.O.-G., R.G.-O., J.P.A.-M.); Novovisión Clinica Dr Gutiérrez, Murcia, Spain (C.V.-C.); and the Clinical \& Experimental Optometry Research Lab, Center of Physics, University of Minho, Braga, Portugal (P.F., J.M.G.-M.).

Inquiries to José M. González-Méijome, CEORLab-Center of Physics (Optometry), School of Science, University of Minho, 4710-057 Gualtar, Braga, Portugal; e-mail: jgmeijome@fisica.uminho.pt
$\mathrm{P}$ HAKIC INTRAOCULAR LENSES (PIOLS) OF DIFFERENT designs and materials have been developed as valuable alternatives to corneal refractive surgery. Although a number of pIOL designs and modifications have been implemented worldwide, the United States Food and Drug Administration has approved only 2 pIOLs: the anterior chamber iris-fixated Artisan/Verisyse pIOL (Ophtec BV, Groningen, The Netherlands/AMO, Santa Ana, California, USA) $)^{1,2}$ that was approved in 2004 and the posterior chamber Visian Implantable Collamer Lens (Visian ICL; STAAR Surgical Company, Nidau, Switzerland) that was approved in December $2005 .^{2}$ The Visian ICL has been reported to be a valuable surgical option to treat various degrees of ametropia. ${ }^{3}$ Two pIOLs currently are undergoing Food and Drug Administration phase 3 clinical trials: the iris-fixated anterior chamber Veriflex/Artiflex (Ophtec BV/AMO) and the anglesupported AcrySof Cachet anterior-chamber pIOL (Alcon Laboratories, Inc, Fort Worth, Texas, USA). Recent studies have shown that the latter pIOL had an acceptable safety profile in patients with myopia. However, potential complications such as long-term endothelial cell loss are a concern. Oversizing of the pIOL can produce excessive vaulting and rapid endothelial cell loss when the internal diameter of the anterior chamber is not well correlated with the white-to-white values. ${ }^{4}$ Elevated intraocular pressure $(\mathrm{IOP})$ is a potential adverse event associated with anterior chamber pIOLs. ${ }^{5}$ The Visian ICL is a posterior chamber pIOL manufactured by Staar Surgical Company and has been reported as a valuable surgical option to treat ametropia of various refractive ranges. ${ }^{2}$

Recently, a new type of Visian ICL has been developed: the Visian ICL V4c with CentraFLOW technology. A central artificial hole called the KS-AquaPORT was added to the center of the ICL optic to improve aqueous humor circulation in the eye. This new development eliminates the need for a preoperative peripheral laser iridotomy or intraoperative peripheral iridectomy, which simplifies the surgical procedure and significantly reduces the complications associated with iridotomy such as hyphema, inflammation, or vitreous and rhegmatogenous retinal detachment. ${ }^{6-9}$ Moreover, uneventful preoperative laser iridotomy may develop a postoperative pupillary block if residual viscoelastic blocks the normal aqueous humor flow or because of closure of a previously patent 
iridotomy. However, it is necessary to evaluate the impact of this new surgical approach on elevations in IOP in the immediate and intermediate postoperative periods, which depend on the physical relationship between the IOL implant, the crystalline lens, and the iris, among other factors.

Earlier studies have evaluated the distance between the ICL and the crystalline lens using ultrasound biomicroscopy ${ }^{10}$ Scheimpflug photography, ${ }^{11}$ or the first generations of spectral-domain optical coherence tomography (OCT) technology. ${ }^{12}$ New Spectralis OCT (Heidelberg Engineering, Heidelberg, Germany) with an anterior segment module offers image acquisition of the anterior chamber and provides high-resolution measurements of the distance between the posterior ICL surface and the anterior crystalline lens pole. This distance, referred to as vault, is the most important issue involved in ICLinduced complications, such as pupillary block ${ }^{13}$ and cataract formation. ${ }^{14}$ Ongoing concerns exist about the risk of late-onset cataract formation, presumably resulting from direct physical contact between the ICL and the crystalline lens, and disruptions in aqueous flow that interfere with lens nutrition and cause metabolic disturbances in the crystalline lens. ${ }^{15}$ The new Visian ICL V4c with CentraFLOW was developed to eliminate such disadvantages. The purpose of the current study was to compare the clinical outcomes with the Visian ICL V4c design with CentraFLOW technology without the need for a peripheral iridotomy with the conventional model V4b.

\section{METHODS}

THE STUDY PROTOCOL WAS REVIEWED AND APPROVED BY the Ethics Committee of Hospital Universitario Morales Meseguer, Murcia, Spain, and was in agreement with the standards of clinical practice and national and international guidelines. Following the tenets of the Declaration of Helsinki, patients were informed about the surgical details and provided written informed consent.

This study included 17 eyes of 9 patients implanted with the Visian ICL V4b model and 18 eyes of 10 patients implanted with the new Visian ICL V4c model at Clínica Novovisión Dr Ramón Gutiérrez, Murcia, Spain, from September 2011 through April 2012. Complete ophthalmologic examinations were performed that included manifest and cycloplegic refractions, keratometry and corneal topography with the Pentacam (Oculus Optikgeräte $\mathrm{GmbH}$, Wetzlar, Germany), Goldmann applanation tonometry, and binocular indirect ophthalmoscopy through dilated pupils.

The inclusion criteria for ICL implantation were age between 20 and 45 years, a stable refractive error during the previous 18 months, and a preoperative spectacle spherical power between -3 and -15 diopters (D). The exclusion criteria included anterior chamber depth of less
TABLE. Preoperative Patient Demographics in Eyes Undergoing Visian Implantable Collamer Lens Implantation with CentraFLOW (ICLV4c) and without CentraFLOW (ICL v4b)

\begin{tabular}{lccc}
\hline Parameter & ICL V4b & ICL V4c & $P$ Value \\
\hline No. of eyes & 17 & 18 & \\
Gender & & & \\
Male & $8(47 \%)$ & $3(17 \%)$ & \\
Female & $9(52 \%)$ & $15(83 \%)$ & \\
Age (y) & $32.6 \pm 7.2$ & $34.5 \pm 9.5$ & .48 \\
Range & 26 to 45 & 26 to 50 & \\
Manifest refraction (D) & & & \\
Sphere & $-7.95 \pm 3.77$ & $-7.68 \pm 3.7$ & .938 \\
$\quad$ Range & -3.25 to $-15.25-1.75$ to -17.25 & \\
Cylinder & $-1.51 \pm 1.13$ & $-1.81 \pm 1.33$ & .578 \\
Range & 0.00 to -3.50 & -0.50 to -5.50 & \\
ACD (mm) & $3.43 \pm 0.37$ & $3.25 \pm 0.18$ & .09 \\
Range & 2.8 to 3.91 & 2.98 to 3.52 & \\
WTW (mm) & $12.15 \pm 0.75$ & $11.96 \pm 0.4$ & .68 \\
Range & 11.09 to 13.60 & 11.25 to 12.63 & \\
ICL design & & & \\
ICM & $9(53 \%)$ & $11(61 \%)$ & \\
TICM & $8(47 \%)$ & $7(39 \%)$ & \\
\hline
\end{tabular}

$\mathrm{ACD}=$ anterior chamber depth; $\mathrm{D}=$ diopter; $\mathrm{ICL}=$ Implantable Collamer Lens; ICM = ICL for myopia; TICM = lens for myopia with astigmatism; WTW = white-to-white distance.

Data are mean \pm standard deviation unless otherwise indicated.

than $2.8 \mathrm{~mm}$, a history of glaucoma or ocular inflammation, pre-existing corneal disease, and endothelial cell density of less than 2500 cells $/ \mathrm{mm}^{2}$

- IMPLANTABLE COLLAMER LENS SELECTION AND CHARACTERISTICS: The one-piece ICL pIOL is made of collamer, a hydrophilic material composed of collagen and poly-hydroxyethyl methacrylate-based copolymer. The lens is available in spherical powers ranging from -3.0 to $-23.0 \mathrm{D}$ and astigmatic corrections of 1.0 to $6.0 \mathrm{D}$. The lens size was defined using the manufacturer calculator software according to the horizontal white-towhite distance obtained by partial coherence interferometry (IOLMaster; Carl Zeiss Meditec, Jena, Germany).

Two subsequent groups were formed with the last ICL V4b model for myopic correction and the first ICL V4c model implanted. Patients scheduled for intraocular refractive surgery from September 2011 through May 2012 were enrolled in this trial. The preoperative demographics of the study population are shown in the Table.

- VAULT ASSESSMENT WITH SPECTRAL-DOMAIN OPTICAL COHERENCE TOMOGRAPHY: A Spectralis SDOCT (Heidelberg Engineering, Heidelberg, Germany) with the anterior segment module was used to acquire images of the anterior chamber; a detailed view of the 

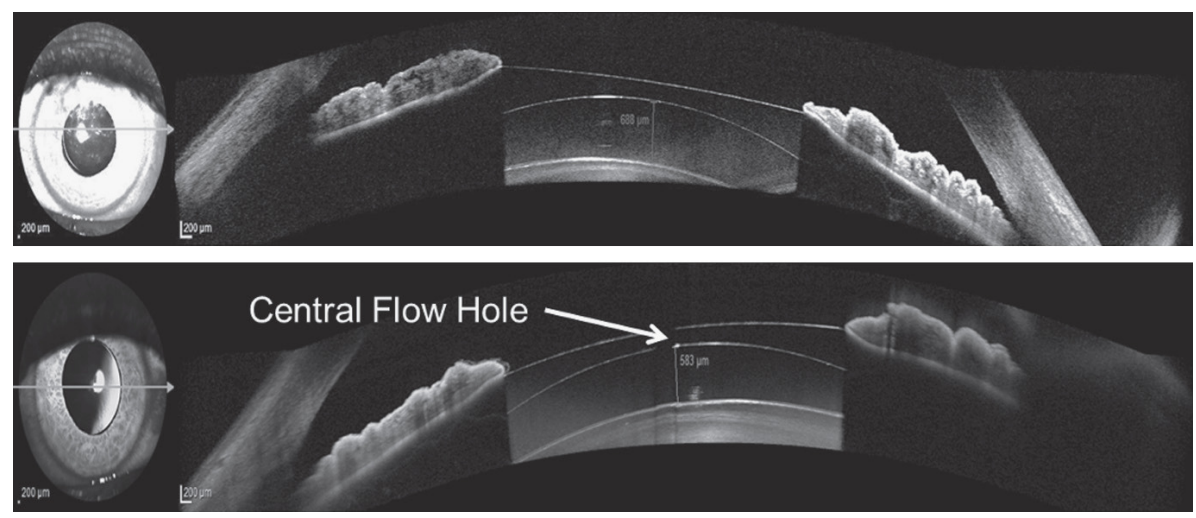

FIGURE 1. Representative Spectralis anterior segment optical coherence tomography images of the distance between the Implantable Collamer Lens (ICL; STAAR Surgical Company) and the anterior lens surface (vault) for the ICL (Top) without a central hole and (Bottom) with CentraFLOW.

ICL and its relationship with intraocular structures was obtained at the 3-month visit (Figure 1). The same observer performed all OCT examinations and vault measurements to determine accurately the implant position in relation to the crystalline lens. OCT was performed under low light conditions, and no pharmacologic agents were used. A fixation light was used for the fellow eye to maintain a fixed amount of accommodation.

- SURGICAL TECHNIQUE: Two neodymium:yttriumaluminum-garnet laser iridotomies were created at the 10 - and 2-o'clock positions 2 weeks before surgery in the ICL V4b group. No iridotomies were performed in the ICL V4c group.

The same surgeon (R.G.-O.) performed all surgeries uneventfully under topical anesthesia (1\% lidocaine). After creating a 3.2-mm clear corneal incision at the steepest meridian, the ICL was inserted with an injector (STAAR Surgical Co.) into the anterior chamber that previously was filled with sodium hyaluronate viscoelastic (Provisc; Alcon Laboratories, Inc). The footplates were placed behind the iris using a fine spatula. Viscoelastic was replaced by balanced salt solution. The wound was closed by stromal hydration after $1 \mathrm{mg}$ cefuroxime sodium was injected into the anterior chamber. ${ }^{16}$ The postoperative therapy included topical moxifloxacin hydrochloride (Vigamox; Alcon Laboratories Inc) 4 times daily for 1 week and dexamethasone (Dexafree; Thea Laboratories, Barcelona, Spain) tapered over 5 weeks.

Postoperative examinations of the uncorrected distance visual acuity, best-corrected distance visual acuity (CDVA), manifested refraction, Goldmann applanation tonometry, and anterior chamber OCT vault were performed on day 1 , week 1 , and months 1 and 3 . A subjective evaluation of the presence of halos and dysphotopsia was carried out by asking the patients and recorded as yes or no.

- STATISTICAL ANALYSIS: The Snellen VA was converted to the logarithm of the minimal angle of resolution values.
Statistical analysis was performed using SPSS for Windows software version 19.0 (SPSS, Inc, Chicago, Illinois, USA). Descriptive statistics were obtained. The normality of data distribution of the measured outcomes was tested using the Shapiro-Wilk test. For all statistical tests, a $P$ value of less than .05 was considered significant. The independent samples Mann-Whitney $U$ test was used to compare the postoperative visual function between the 2 groups. Statistical comparisons between the preoperative and postoperative IOP values in each group were carried out using a repeated measures analysis of variance with post hoc correction (Bonferroni) for multiple comparisons. The independent samples $t$ test was used to compare the postoperative IOP values and vault readings between the groups.

\section{RESULTS}

- REFRACTIVE AND VISUAL OUTCOMES: The postoperative spherical equivalent refractions were $-0.2 \pm 0.4$ and $-0.3 \pm 0.5$ for the $\mathrm{V} 4 \mathrm{~b}$ and $\mathrm{V} 4 \mathrm{c}$ groups, respectively. At 3 months, the respective mean uncorrected distance visual acuity was $-0.09 \pm 0.12$ logarithm of the minimal angle of resolution units in the V4b group and $-0.07 \pm$ 0.11 logarithm of the minimal angle of resolution units in the V4c group $(P=.43) ; 15(88.2 \%)$ and $17(94.4 \%)$ of the eyes, respectively, achieved the same or superior uncorrected distance visual acuity after surgery compared with the preoperative CDVA. Only $1(5.9 \%)$ eye in the V $4 b$ group did not achieve the desired target refraction; all patients in the $\mathrm{V} 4 \mathrm{c}$ group were within $\pm 0.5 \mathrm{D}$ of the target refraction.

Overall, the CDVA improved at all follow-up visits in $9(52.9 \%)$ of the V4b group and $8(44.4 \%)$ of the V4c group. The CDVA was similar in $6(35.3 \%)$ of the V4b group and 9 $(50.0 \%)$ of the V4c group. A decrease of CDVA occurred in only $2(11.8 \%)$ of eyes in the V4b group and in $1(5.6 \%)$ eye in the $\mathrm{V} 4 \mathrm{c}$ group. There were no significant $(P=.81)$ 


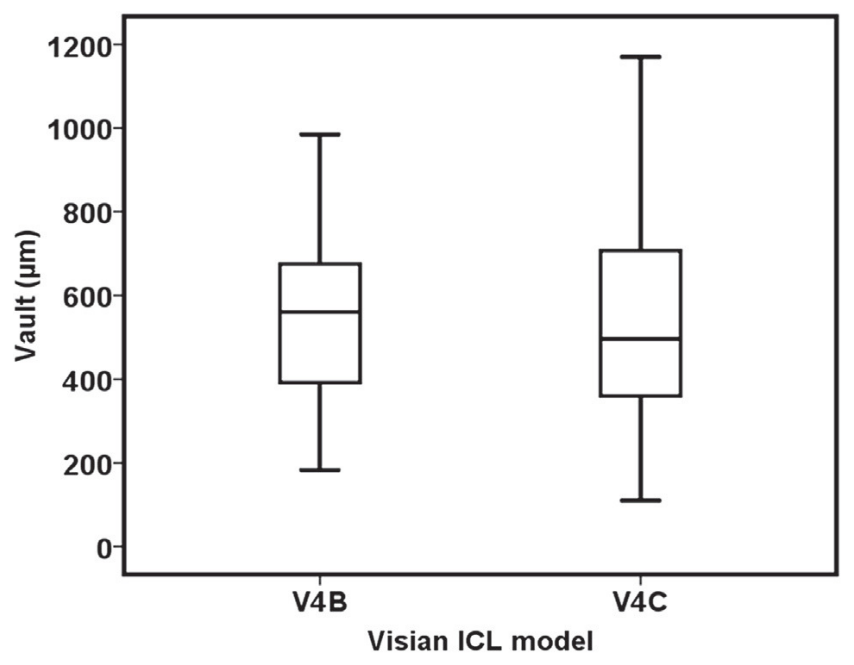

FIGURE 2. Box-and-whisker plot showing the distance between the Implantable Collamer Lens (ICL; STAAR Surgical Company) without a central hole (ICL V4b) and with CentraFLOW (ICL V4c). At 3 months, the mean vault was $557 \pm$ $224 \mu \mathrm{m}$ in the ICL V4b group and $528 \pm 268 \mu \mathrm{m}$ in V4c group with no significant differences between groups $(P=.73)$.

differences between groups. A subjective assessment of patient satisfaction showed that 5 patients reported halos in the $\mathrm{V} 4 \mathrm{c}$ group, which decreased 1 month after surgery.

- VAULT MEASUREMENT: At 3 months, the mean vault was $557 \pm 224.4 \mu \mathrm{m}$ (95\% confidence interval, 442 to $672 \mu \mathrm{m})$ in the V4b group and $528 \pm 268 \mu \mathrm{m}(95 \%$ confidence interval, 354 to $635 \mu \mathrm{m})$ in the $\mathrm{V} 4 \mathrm{c}$ group; this difference did not reach significance $(P=.73$; Figure 2$)$. There was no correlation between age and vault value in the V4b group (Pearson $\rho,-0.244 ; P=.16$ ) or the V4c group (Pearson $\rho,-0.16 ; P=.95$ ).

- INTRAOCULAR PRESSURE MEASUREMENT: We observed a mild and transient increase in IOP during the first month; however, no chronically elevated IOP levels or pupillary block were observed in any group. The mean preoperative $\mathrm{IOP}$ values were $11.5 \pm 2.8 \mathrm{~mm} \mathrm{Hg}$ in the V4b group and $11.9 \pm 2.7 \mathrm{~mm} \mathrm{Hg}$ in the $\mathrm{V} 4 \mathrm{c}$ group $(P=.66)$. At 3 months, the mean IOPs were $12.4 \pm 1.8 \mathrm{~mm} \mathrm{Hg}$ and $13.8 \pm 2.2 \mathrm{~mm}$ $\mathrm{Hg}$ in the $\mathrm{V} 4 \mathrm{~b}$ and $\mathrm{V} 4 \mathrm{c}$ group, respectively (Figure 3 ). There were no significant differences in the mean IOP within each group during the follow-up period $(F=.49, P=.64)$ or between groups at any follow-up visits $(P>.05$, for all comparisons).

\section{DISCUSSION}

ICL IMPLANTS EFFECTIVELY CORRECT MODERATE TO HIGH myopia, which has been widely reported in the literature. ${ }^{1,2}$ The current study found comparable visual and refractive

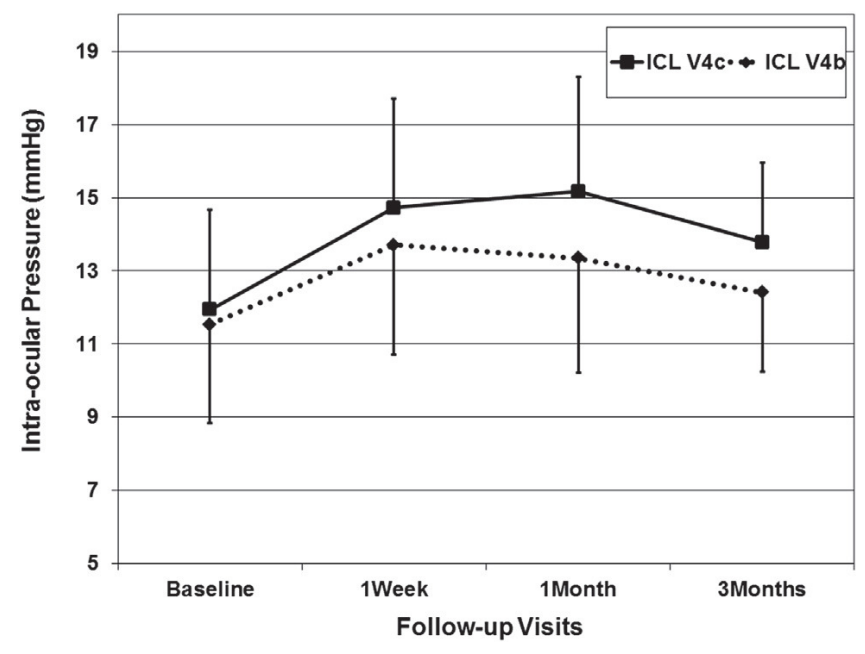

FIGURE 3. Graph showing intraocular pressure (IOP) levels before and after implantation of the Implantable Collamer Lens (ICL; STAAR Surgical Company) without a central hole (ICL V4b) and with CentraFLOW (ICL V4c). At 3 months, the mean IOP was $12.4 \pm 1.8 \mathrm{~mm} \mathrm{Hg}$ and $13.8 \pm$ $2.2 \mathrm{~mm} \mathrm{Hg}$ in the ICL V4b and ICL V4c groups, respectively. There were no significant differences in the mean IOP within each group during the follow-up period $(F=0.49, P=.64)$ or between groups at any time during the follow-up period ( $P>$.05, for all comparisons).

outcomes between both ICLs models, with no differences in postoperative refraction or accuracy of the refractive results between the 2 groups throughout the 3 -month follow-up period. The efficacy of the procedure was slightly better in the V4c group, with $94.4 \%$ and $88.2 \%$ of eyes having an uncorrected visual acuity equal or superior to the preoperative CDVA in the V4c and V4b group, respectively.

Regarding the optical performance of the new ICL with CentraFLOW, although we have not evaluated the change in the higher-order aberrations (HOAs) between the 2 groups, we recorded temporary halos in the $\mathrm{V} 4 \mathrm{c}$ group based on the subjective patient assessment. Induced negative spherical aberration and increased trefoil have been reported after ICL implantation. ${ }^{17}$ Other studies have reported that the ICL is at least as effective as excimer laser intervention even in patients with a low spherical equivalent refraction, with significantly lower induced spherical aberrations after ICL implantation compared with laser in situ keratomileusis. ${ }^{18}$ Subjective visual symptoms, such as glare or halo, have been reported to be common in the early postoperative period in conventional ICLimplanted eyes. Regarding the new ICL with the central hole, the presence of the central hole may deteriorate the performance of the ICL to some extent and may induce glare or halos. An animal model ${ }^{19}$ and in vitro optical simulation studies ${ }^{20}$ have reported small and clinically negligible differences between both types of ICLs. In the 
current study, patient reports of halos in the V4c group significantly decreased 1 month after surgery; however, scattering and HOAs should be investigated in future studies. The complaints about halos have been transient in these patients, suggesting an adaptation process to the presence of the ICL. Considering the small sample size, it is impossible to evaluate potential relationships between the optical power of the lens or potential positioning issues, which may help to explain these photic phenomena. Although HOAs might have some correlation with halos as we found in patients who underwent laser in situ keratomileusis, ${ }^{21}$ weak correlations were found in those patients whose optical quality clearly was altered by corneal ablation, and we did not expect that this would explain the phenomena in patients with an ICL in whom HOAs with previous types of lenses have caused much fewer changes compared with ablative procedures. As a result, we should not expect significant changes in contrast sensitivity function considering the good optical quality associated with ICL implantation. ${ }^{17,18}$ Nevertheless, the current data did not allow us to ignore the potential impact of HOAs on the development of halos.

Conventional ICL implantation requires preoperative laser iridectomies or intraoperative peripheral iridectomy to prevent pupillary block. Neodyium:yttrium-aluminumgarnet laser iridotomy carries the potential risk of intraocular complications such as hyphema, marked inflammation, elevated IOP, chronic pigment dispersion, posterior synechiae, and vitreous and rhegmatogenous retinal detachments. ${ }^{22,23}$ Significant reductions of these complications may be expected with the new ICL with the central hole, which eliminates the need for neodyium:yttriumaluminum-garnet iridotomy or peripheral iridectomy before implantation of the ICL and the potential complications associated with these procedures. Acute pupillary block, which frequently is associated with inadequate preoperative iridotomies, ${ }^{22}$ currently is managed by enlargement of existing iridotomies or creation of additional peripheral iridectomies and may be avoided using the new $\mathrm{V} 4 \mathrm{c}$ design.

Development of anterior subcapsular opacities and clinically relevant cataract remain major concerns in ICL implantation. The main theories of the cause of this cataract development are the absent or lower vault and the tendency of the vault to decrease over time, causing constant or intermittent contact between the ICL and crystalline lens. Studies that evaluated the pathophysiologic features of anterior subcapsular cataracts secondary to ICL implantation confirmed that contact with or close proximity of the phakic lens to the crystalline capsule might have disrupted the aqueous flow, which may have led to metabolic changes and transformations within the crystalline lens. ${ }^{15}$ The ideal postoperative vault must create a space over the entire anterior crystalline lens surface, with the recommended amount of vault equal to
1.0 to 1.5 central corneal thicknesses on slit-lamp examination. ${ }^{24}$ Poor vault $(<250 \mu \mathrm{m})$ increases cataract development, but excessive vault $(>750 \mu \mathrm{m})$ can cause angle closure, pupillary block, and glaucoma. Vaulting of the ICL is affected by the ICL shape (ie, the V4 model was an improved version of the flatter V3 previous design), ${ }^{6}$ white-to-white measurement, ${ }^{25}$ and age. ${ }^{11}$ To demonstrate the safety of the new ICL design, it was important to determine if vault values were comparable with the classical ICL model. With spectral-domain OCT, 2 dimensional and high-resolution images of the anterior ocular segment can be obtained rapidly and with noncontact procedures. ${ }^{12}$ It has been useful to elucidate the correlation between the ICL and adjacent intraocular structures and helpful to evaluate surgical procedures and their potential complications. ${ }^{26}$ Regarding vaulting, the current study found no significant differences between the ICLs, which led us to believe that both lenses have a similar anatomic interaction with the intraocular structures. This finding also may indicate that the central hole contributes to improved circulation of the aqueous humor to the anterior surface of the crystalline lens and therefore prevents cataract formation. However, the short follow-up in this small number of the patients is insufficient for detecting such complications; longer follow-up with more patients is required to obtain longer-term safety data for the new ICL design.

We found no significant increases in IOP (including pupillary block) throughout the 3 -month observation period or significant differences between the groups. Thus, despite the absence of peripheral iridotomy, normal aqueous flow seems to occur as a result of the central hole in the new ICL V4c, which maintained in physiologic IOP levels during the follow-up period in both groups. Most studies have reported increased IOP levels after ICL implantation, suggested to be a potential cause of pupillary block, pigment dispersion, and steroid use. ${ }^{22}$ Spectraldomain OCT images showed ICL-iris contact in both groups, but the authors did not observe the potential pigmentary dispersion previously observed with this refractive procedure. Other authors suggested that the soft hydrophilic surface of the posterior chamber phakic IOL is similar to the anterior capsule of the crystalline lens and may prevent a mechanical loss of pigment. ${ }^{27}$

In summary, the current study showed the 3 -month safety and efficacy of the new ICL V4c with CentraFLOW design. Although longer observation in a large number of patients is required to assess the long-term safety of this surgical procedure, clinically, the new model did not differ in its intraoperative properties or postoperative follow-up from those of the conventional ICL. We concluded that the visual results, vault measurements, and IOP levels were comparable in both groups. Another advantage was that iridotomies were not needed because of the new ICL $\mathrm{V} 4 \mathrm{c}$ design. 
ALL AUTHORS HAVE COMPLETED AND SUBMITTED THE ICMJE FORM FOR DISCLOSURE OF POTENTIAL CONFLICTS OF INTEREST and none were reported. Supported in part by a grant from the Spanish Ministry of Health, Instituto Carlos III, Red Temática de Investigación Cooperativa en Salud Patología Ocular del Envejecimiento, Calidad Visual y Calidad de Vida, Subproyecto de Calidad Visual, Madrid, Spain (RD07/0062). Involved in Design and conduct of study (A.H.-E., C.V.-C., R.G.-O., P.F., J.G.-M.); Collection, management, analysis, and interpretation of data (A.H.-E., J.G.-M., P.F.); and Preparation, review, or approval of manuscript (A.H.-E., A.O.-G., C.V.-C., R.G.-O., J.-A., P.F., J.G.-M.).

\section{REFERENCES}

1. Stulting RD, John ME, Maloney RK, Assil KK, Arrowsmith PN, Thompson VM, U.S. Verisyse Study Group. Three-year results of Artisan/Verisyse phakic intraocular lens implantation. Results of the United States Food And Drug Administration clinical trial. Ophthalmology 2008;115(3): 464-472.

2. Pop M, Payette Y. Initial results of endothelial cell counts after Artisan lens for phakic eyes: an evaluation of the United States Food and Drug Administration Ophtec Study. Ophthalmology 2004;111(2):309-317.

3. Sanders DR, Doney K, Poco M. United States Food and Drug Administration clinical trial of the Implantable Collamer Lens (ICL) for moderate to high myopia: three-year followup. Ophthalmology 2004;111(9):1683-1692.

4. Pechméja J, Guinguet J, Colin J, Binder PS. Severe endothelial cell loss with anterior chamber phakic intraocular lenses. J Cataract Refract Surg 2012;38(7):1288-1292.

5. Alió JL, de la Hoz F, Pérez-Santonja JJ, Ruiz-Moreno JM, Quesada JA. Phakic anterior chamber lenses for the correction of myopia: a 7-year cumulative analysis of complications in 263 cases. Ophthalmology 1999;106(3): 458-466.

6. Gonvers M, Bornet C, Othenin-Girard P. Implantable contact lens for moderate to high myopia: relationship of vaulting to cataract formation. J Cataract Refract Surg 2003; 29(5):918-924.

7. Siam GA, de Barros DS, Gheith ME, et al. Post-peripheral iridotomy inflammation in patients with dark pigmentation. Ophthalmic Surg Lasers Imaging 2008;39(1):49-53.

8. Kumar N, Feyi-Waboso A. Intractable secondary glaucoma from hyphema following YAG iridotomy. Can J Ophthalmol 2005;40(1):85-86.

9. Kraemer C, Gramer E. Posterior synechiae after Nd:YAG laser iridotomy. A clinical study. Ophthalmologe 1998;95(9): 625-632.

10. Trindade F, Pereira F, Cronemberger S. Ultrasound biomicroscopic imaging of posterior chamber phakic intraocular lens. J Refract Surg 1998;14(5):497-503.

11. Lindland A, Heger H, Kugelberg M, Zetterstrom C. Vaulting of myopic and toric Implantable Collamer Lenses during accommodation measured with Visante optical coherence tomography. Ophthalmology 2010;117(6):1245-1250.

12. Alfonso JF, Lisa C, Palacios A, Fernandes P, GonzélezMéijome JM, Montés-Micó R. Objective vs subjective vault measurement after myopic implantable collamer lens implantation. Am J Ophthalmol 2009;147(6): 978-983.

13. Bylsma SS, Zalta AH, Foley E, Osher RH. Phakic posterior chamber intraocular lens pupillary block. J Cataract Refract Surg 2002;28(12):2222-2228.
14. Sanders DR. Anterior subcapsular opacities and cataracts 5 years after surgery in the Visian implantable collamer lens FDA trial. J Refract Surg 2008;24(6):566-570.

15. Khalifa YM, Moshirfar M, Mifflin MD, Kamae K, Mamalis N, Werner L. Cataract development associated with collagen copolymer posterior chamber phakic intraocular lenses: clinicopathological correlation. J Cataract Refract Surg 2010; 36(10):1768-1774.

16. Endophthalmitis Study Group. European Society of Cataract $\&$ Refractive Surgeons. Prophylaxis of postoperative endophthalmitis following cataract surgery: results of the ESCRS multicenter study and identification of risk factors. J Cataract Refract Surg 2007;33(6):978-988.

17. Kim SW, Yang H, Yoon G, et al. Higher-order aberration changes after Implantable Collamer Lens implantation for myopia. Am J Ophthalmol 2011;151(4):653-662.

18. Sanders DR. Matched population comparison of the Visian Implantable Collamer Lens and standard LASIK for myopia of -3.00 to -7.88 diopters. J Refract Surg 2007;23(6):537-553.

19. Shiratani T, Shimizu K, Fujisawa K, Uga S, Nagano K, Murakami Y. Crystalline lens changes in porcine eyes with implanted phakic IOL (ICL) with a central hole. Graefes Arch Clin Exp Ophthalmol 2008;246(5):719-728.

20. Uozato H, Shimizu K, Kawamorita T, Ohmoto F. Modulation transfer function of intraocular collamer lens with a central artificial hole. Graefes Arch Clin Exp Ophthalmol 2011; 249(7):1081-1085.

21. Villa C, Gutiérrez R, Jiménez JR, González-Méijome JM. Night vision disturbances after successful LASIK surgery. $\mathrm{Br}$ J Ophthalmol 2007;91(8):1031-1037.

22. Fernandes P, González-Méijome JM, Madrid-Costa D, FerrerBlasco T, Jorge J, Montés-Micó R. Implantable collamer posterior chamber intraocular lenses: a review of potential complications. J Refract Surg 2011;27(10):765-776.

23. de Silva DJ, Day AC, Bunce C, Gazzard G, Foster PJ. Randomised trial of sequential pretreatment for $\mathrm{Nd}$ :YAG laser iridotomy in dark irides. Br J Ophthalmol 2012;96(2):263-266.

24. Sanders DR, Vukich JA, Doney K, Gaston M. U.S. Food and Drug Administration clinical trial of the Implantable Contact Lens for moderate to high myopia. Ophthalmology 2003;110(2):255-266.

25. Alfonso JF, Fernández-Vega L, Lisa C, Fernandes P, Jorge J, Montés Micó R. Central vault after phakic intraocular lens implantation: correlation with anterior chamber depth, white-to-white distance, spherical equivalent, and patient age. J Cataract Refract Surg 2012;38(1):46-53.

26. Malyugin BE, Shpak AA, Pokrovskiy DF. Accommodative changes in anterior chamber depth in patients with high myopia. J Cataract Refract Surg 2012;38(8):1403-1407.

27. Assetto V, Benedetti S, Pesando P. Collamer intraocular contact lens to correct high myopia. J Cataract Refract Surg 1996;22(5):551-556. 


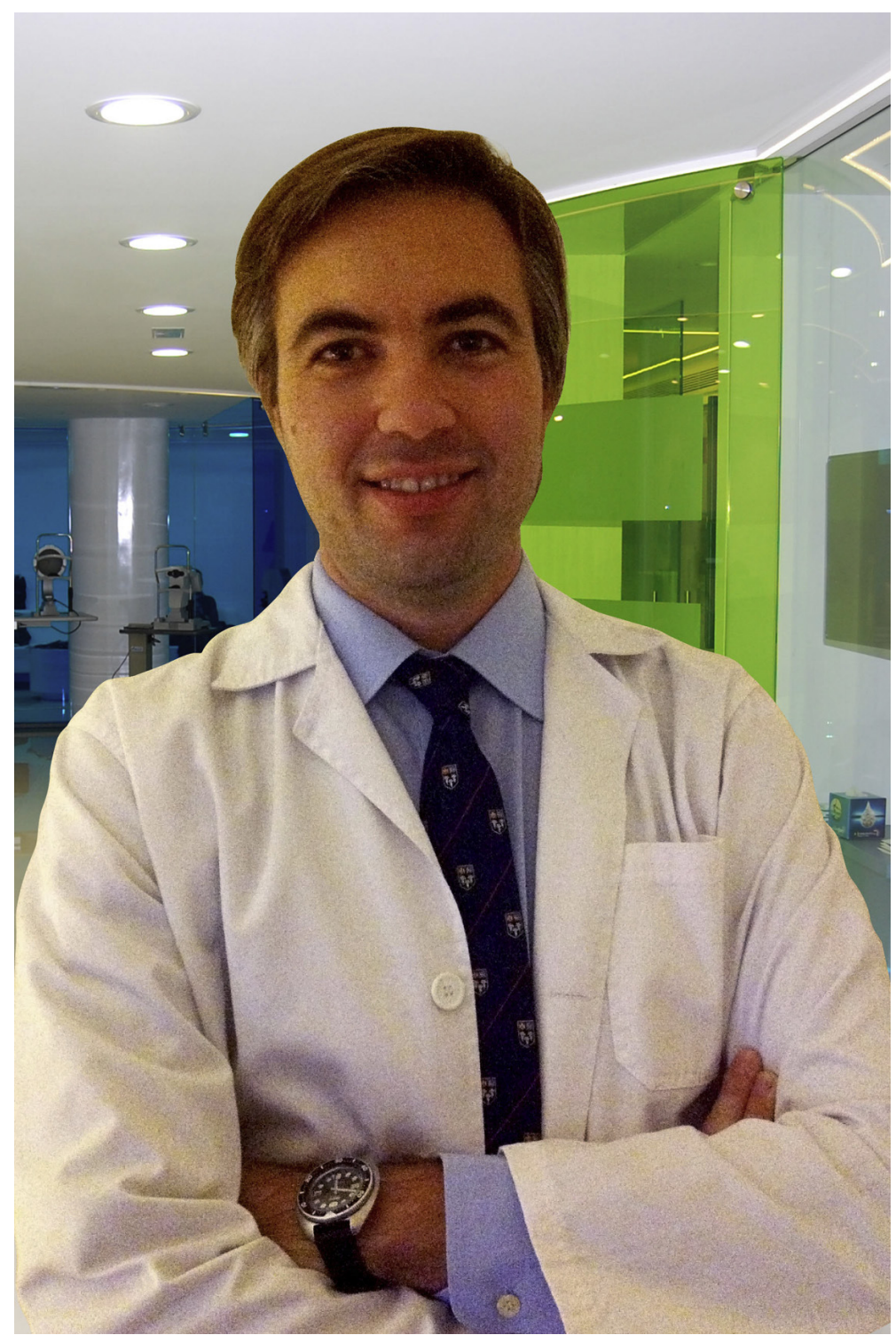

\section{Biosketch}

Alejandro Higueras-Esteban, MD, is an Assistant Professor of the Department of Ophthalmology, at Murcia University School of Medicine. He received his MD from Granada University School of Medicine in 2004. He did his Ophthalmology residency at Reina Sofia de Murcia Hospital, and is currently working as a consultant at the Refractive surgery department, Clínica Novovisión Murcia, Spain. 